\title{
THE ADVANCED X-RAY ASTROPHYSICS FACILITY
}

\author{
M.C. WEISSKOPF \\ Space Science Laboratory, NASA Marshall Space Flight Center, Alabama 35812
}

\begin{abstract}
The Advanced X-Ray Astrophysics Facility (AXAF) is a remarkable and unique scientific endeavor. It will be a space-based earth orbiting X-ray observatory, designed to address fundamental questions in astronomy and astrophysics. It is remarkable in that it represents enormous advances in observational capability. It is unique in that it will be the best means of obtaining high resolution, spectrally resolved X-ray images of astronomical objects for the rest of this century and beyond.
\end{abstract}

\section{Introduction}

Before the onset of the space age our view of the universe was restricted to those wavelength bands in which the earth's atmosphere was essentially transparent. Our current ability to place sophisticated instrumentation in orbit has opened new windows for studying the cosmos. In particular, X-ray astronomy has become a major discipline of astronomy. X-ray observations, which began by utilizing sounding rockets in the early 60 's, have revealed a universe involving violent and energetic phenomena wherein particles are accelerated to relativistic velocities, wherein we see plasmas at temperatures from $10^{6}$ to $10^{9} \mathrm{~K}$, and where there are magnetic fields stronger than $10^{12}$ Gauss. X-ray observations have discovered such phenomena in wide variety of astronomical settings ranging from normal stars to quasars and have had a profound influence on astronomy, astrophysics and cosmology.

The ubiquity of X-ray emission together with the fact that many of the fundamental physical processes give rise to copious amounts of X-ray emissions are the basis of the scientific importance of the AXAF. The growing partnership between astronomy and physics further supports this importance. The ultimate fate of the universe could be discovered by accurate and unambiguous measurements of the the Hubble constant and the deceleration parameter. The existence of exotic particles predicted by supersymmetric particle theories might be confirmed or ruled out by observing their effects on the hot X-ray emitting gas found in galaxies and clusters of galaxies. The equation of state of matter at nuclear densities can be tested by studying the $\mathrm{X}$-radiation from neutron stars. These investigations, which will be performed with the AXAF, address some of the most fundamental questions in science today. The AXAF will play a crucial role in finding the answers.

AXAF is the successor to NASA's second High Energy Astronomy Observatory, the EINSTEIN, which was flown from 1978-1981, and made significant discoveries. Like EINSTEIN, AXAF will utilize grazing incidence X-ray optics capable of forming X-ray images. AXAF, however, will go far beyond EINSTEIN in capability. $\mathrm{AXAF}$ will have $\approx 10$ times better angular resolution, 100 times better sensitivity for the detection of point sources, and up to 1000 times the sensitivity for high resolution spectroscopy. Using the history of science as a guide, such increases in

Y. Kondo (ed.), Observatories in Earth Orbit and Beyond, 71-79.

(C) 1990 Kluwer Academic Publishers. Printed in The Netherlands. 
sensitivity guarantee that AXAF will make major advances in our understanding of nature.

To accomplish its objectives, AXAF will be built around a large area, high resolution, grazing incidence X-ray telescope, accompanied by a complement of imaging and spectroscopic instruments which can be maintained and/or replaced in orbit. The optics, of Wolter-I design, are formed of parabolic and hyperbolic surfaces of revolution. The overall characteristics of the facility and its first generation instruments are summarized in Table I. A more detailed discussion of the AXAF characteristics may be found in the review paper by Weisskopf (1987).

It is important to note that AXAF's long life of 15 years will contribute to its scientific potential. There will be time for the new theoretical and observational work stimulated by unexpected discoveries to be checked against the phenomena while the phenomena are still open to observation. This type of capability represents an important step for space astronomy.

The AXAF will be open for use by the entire astronomical community, with the majority of observing time and all the archival data set aside for general observers to be selected by peer review. Based on NASA's experience with a number of programs, this also implies substantial international participation.

\section{Galaxy Clusters}

AXAF is critical to progress in astrophysics because it will be the premiere facility for observations in the X-ray region of the electromagnetic spectrum. It is no accident that this portion of the spectrum is so rich in information. This richness is a consequence of the convolution of any extragalactic X-ray source spectrum with the transmission of this flux in the interstellar medium in our galaxy which leads to a peak response in low energy X-rays. The spectral sensitivity of AXAF (0.1-10 keV) thus offers a direct means of probing the universe in a critical wavelength region.

The phenomena that give rise to $\mathrm{X}$-ray emission are of paramount importance in that they involve highly energetic cosmic events and their consequences. These include stellar and galactic explosions, accretion of matter onto gravitational collapsed objects, and high temperature gases in variety settings including clusters of galaxies. Phenomena such as these announce their existence primarily through their X-ray emission and provide us with a different picture of the universe both qualitative and quantitaive, than the one we are used to seeing. As an example, we show in Figure 1 the optical and X-ray view of a cluster of galaxies -A1367. The X-ray emission is dominated by the extended emission coming from a diffuse, hot gas filling the space between the galaxies in the cluster. This gas has a characteristic temperature of about $5 \times 10^{7} \mathrm{~K}$ and therefore radiates primarily at $\mathrm{X}$-ray wavelengths. Visible light images gave no hint as to the existence of this gas; yet the gas itself accounts for about half of the directly observable mass of the cluster and provides important structural information through its density and temperature profiles. Clearly a thorough understanding of the formation, dynamics, and evolution of such systems will be accomplished with AXAF.

AXAF spectra of the hot gas in clusters will allow examination of the heavy element content, already known to be signficant from earlier X-ray observations, 
TABLE I

AXAF characteristics

Weight

Dimensions

Power

Data Rate

Pointing Accuracy

Pointing Stability

Post Facto Aspect Accuracy

Post Facto Aspect Accuracy

Telescope Angular Resolution

Telescope Dimensions

Telescope Geometric Area

Spectral Range

Orbit

Lifetime

Instruments

Low Energy Transmission Gratings

High Energy Transmission Gratings

$C C D$ imager

Microchannel Plate Imager

$X$-ray Calorimeter
$12,000 \mathrm{~kg}$

cylindrical, $14 \mathrm{~m}$ long, $4 \mathrm{~m}$ diameter

2500 watts, orbital average for entire observatory

32 kbps, 24 kbps science

30 arc sec

0.5 arc sec for $10 \mathrm{sec}$

0.5 arc sec (rms diameter) relative

1.0 arc sec ( $1 \sigma$ radius) absolute

0.5 arc sec FWHM

$1.2 \mathrm{~m}$ diameter, $10 \mathrm{~m}$ focal length, $1^{\circ}$ field of view $1700 \mathrm{~cm}^{2}$

$0.1-10 \mathrm{keV}$

$600 \mathrm{~km}$ circular, 28.5 inclination

15 years with on-orbit servicing

record position, energy, and arrival time for

individual photons

cover the bandwidth from $0.1-4.0 \mathrm{keV}$ with resolving power from about 2000 at low energies falling to 100 at $2 \mathrm{keV}$.

cover the bandwidth from $0.5-9.0 \mathrm{keV}$ with resolving power from 1000 at the lower energies falling to 100 at $9 \mathrm{keV}$.

an array of CCD devices with sub arc-second angular resolution, approximately $150 \mathrm{eV}$ energy resolution and an energy-dependent quantum efficiency that averages better than $30 \%$ over most of the $0.1-10.0 \mathrm{keV}$ band width.

A $32^{\prime}$ by $32^{\prime}$ imager with 0.5 arc-second angular resolution.

The device has an energy-dependent efficiency ranging from $20-50 \%(0.1-3 \mathrm{keV})$ and $10-20 \%(3-8 \mathrm{keV})$ and offers a modest spectral resolution of 1 at $1 \mathrm{keV}$.

a spectrometer with $10 \mathrm{eV}$ energy resolution and high quantum efficiency which subtends a 1 arc-minute square field-of-view. 


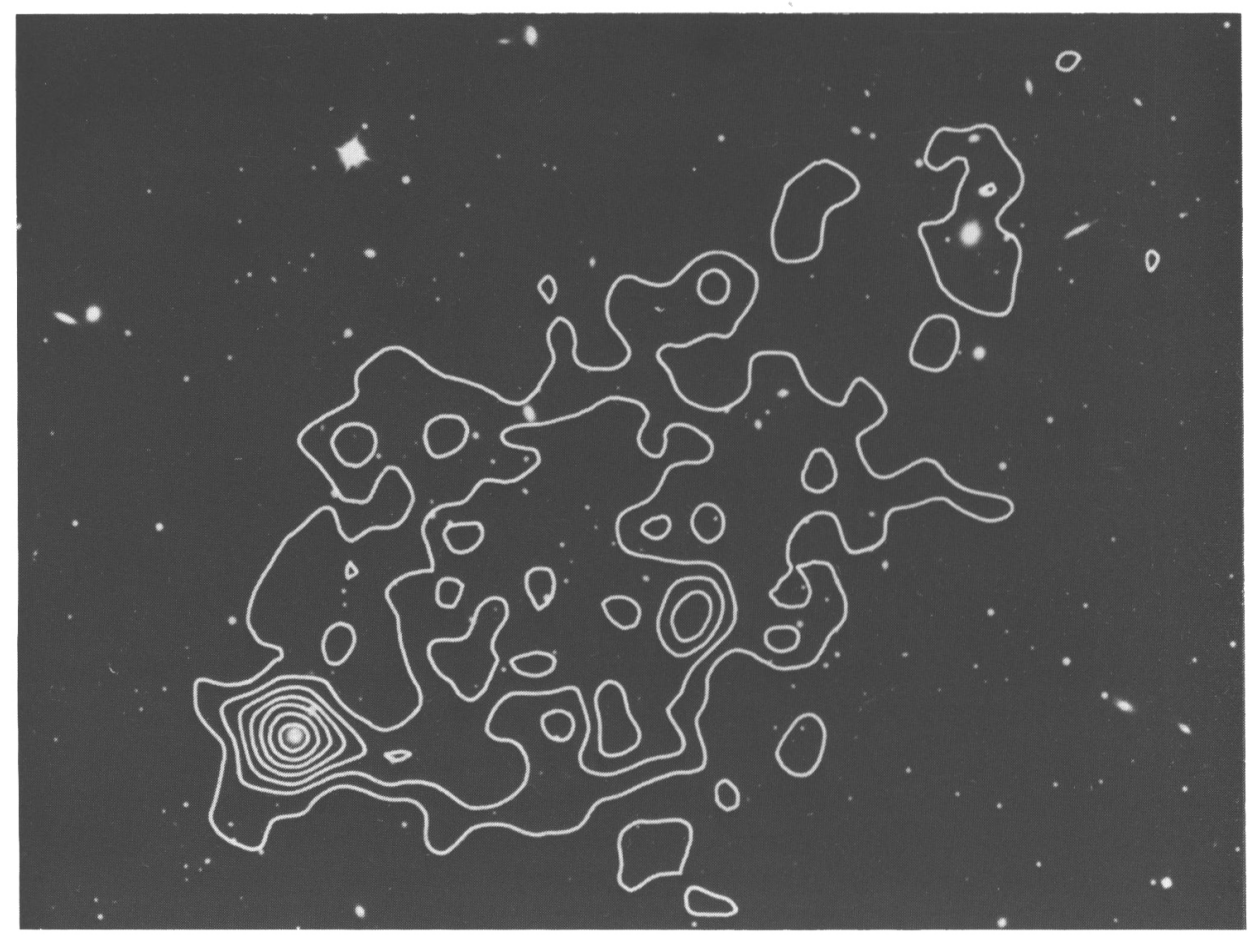

Fig. 1. Optical image and X-ray contours of A1367.

but previously impossible to study in detail due to an inability to combine high angular and high energy resolution. For the nearer clusters AXAF will be able to measure the spatial distribution, temperature and ionization state of the heavyelement content through iron. The potential of such studies is illustrated in Figure 2, the Einstein image of the galaxy M86, a member of the Virgo cluster of galaxies. The extended plume to the upper right indicates a large amount of gas being stripped from the galaxy and added to the cluster gas. AXAF will be able to measure the heavy element content of the plume and compare it to the cluster as a whole to see if the galactic gas is relatively enriched, as would be expected if it had been processed through stars. If all the heavy elements are produced in stars, AXAF studies of more distant, and therefore younger clusters, should uncover an evolutionary pattern. Even then, possible surprises could be provided by the detection of heavy elements produced by a very short-lived generation of massive stars that exploded very early in the cluster development. Such studies, including the detailed X-ray spectroscopy of supernova in our own and nearby galaxies, should cast new light on the sequence of events leading from the Big Bang to the formation of structure in galaxies, stars, and provide essential information about the seed material from which our solar system and indeed we were formed.

An extremely important question that AXAF will address involve understanding how the hot gas, that permeates individual galaxies, as well as clusters, is heated. 


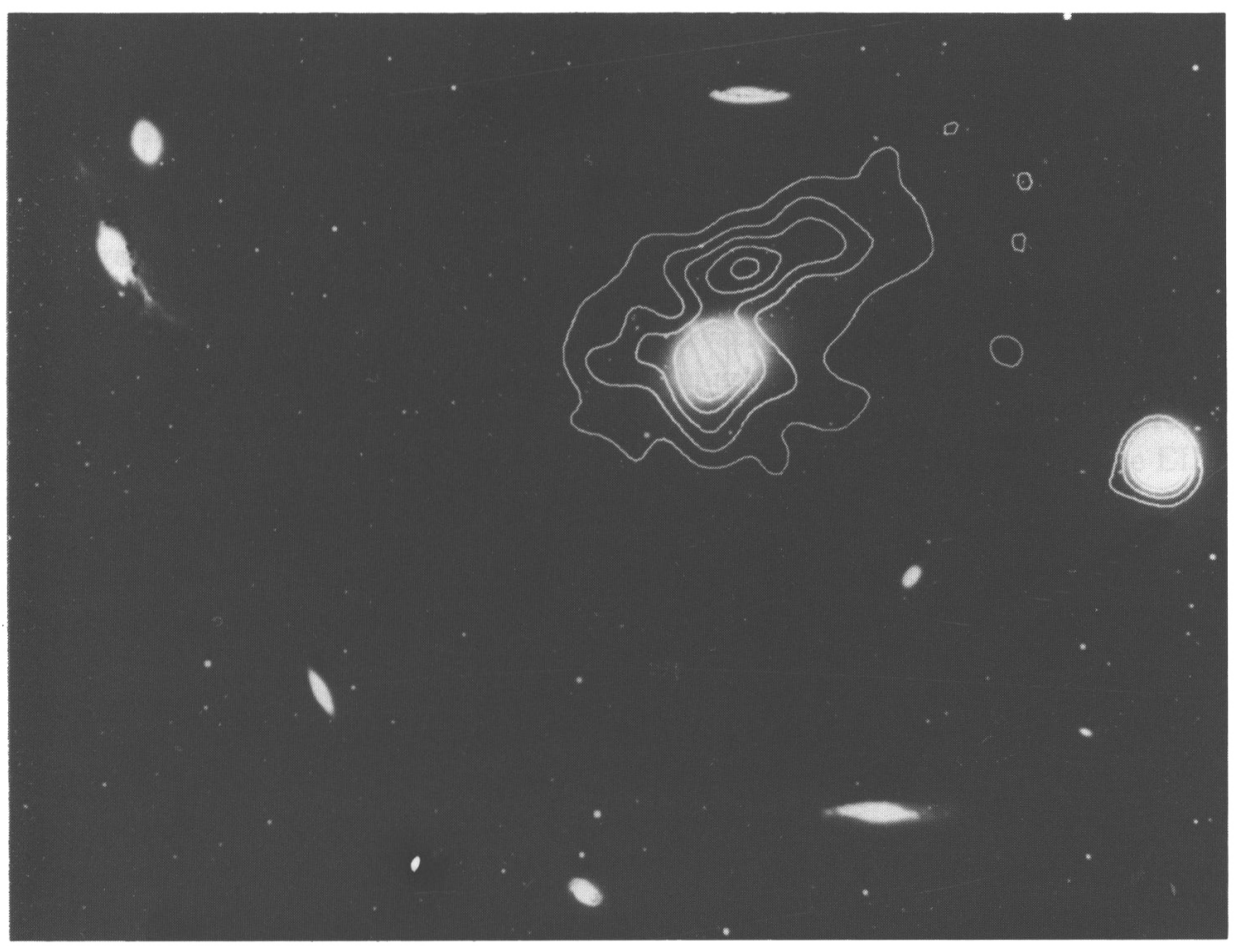

Fig. 2. Optical image and X-ray contours of M86 showing the extended plume.

Further, observations of the manner in which these gases cool through their $\mathrm{X}$ ray emission have important relevance for star formation, which requires a large aggregation of gas to lose enough heat so it can collapse under its own gravity.

\section{Dark Matter}

Observations with AXAF will be particularly well-suited for measuring the distribution of dark matter on various size scales, from stars, to galaxies, to clusters of galaxies, to clusters of clusters or superclusters. Although theories abound, the dark matter has not to date been directly observed, it can be studied through its gravitational effects. The very hot gas (e.g., Figure 1), which can only be seen in X-rays has been observed in many large astronomical systems and is evidently being held in the gravitational grip of dark matter. Careful observation of such gas with AXAF can be used to map out the structure of this gravitational field, which depends on the temperature and mass of the individual dark matter particles. Comparisons between the observed and predicted properties of the structure of the gravitational field can help in evaluating different theories.

On a more speculative level it is feasible that AXAF may directly detect the $\mathrm{X}$-ray emission associated with some of the exotic dark matter candidates such as one-dimensional discontinuities in quantum gauge fields, called cosmic strings. Some 


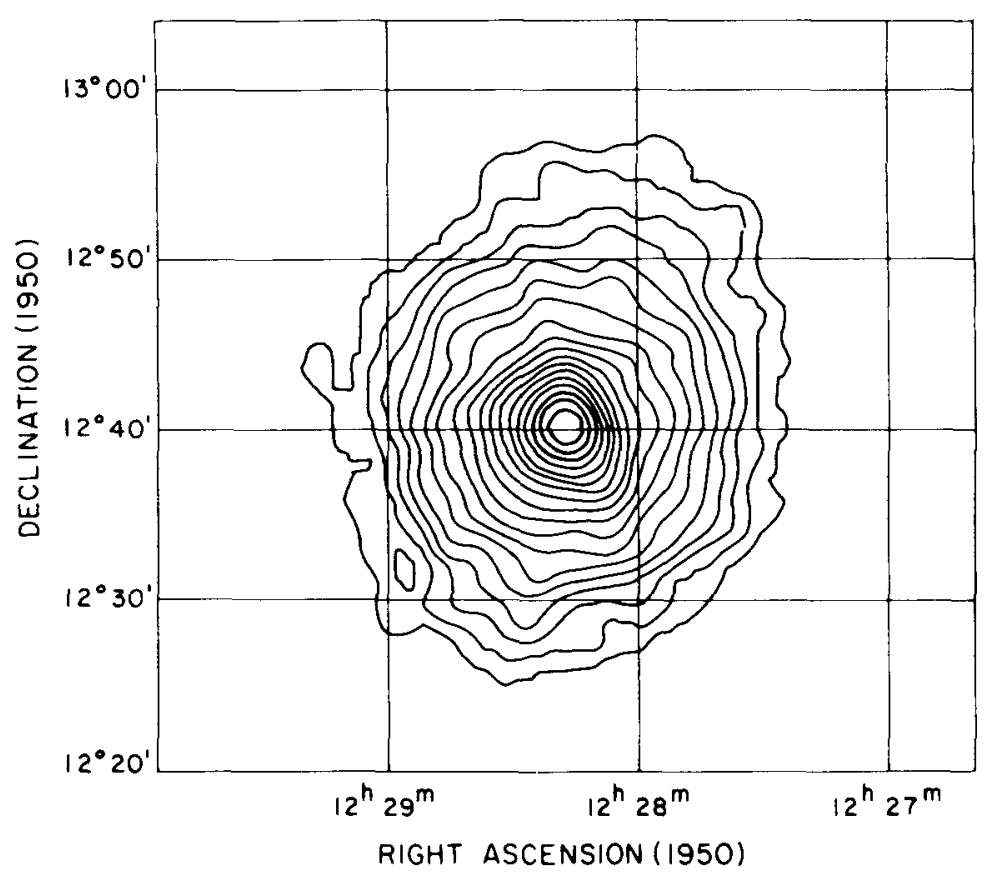

Fig. 3. X-ray contours of the giant ellptical galaxy M87.

new theories in particle physics predict that these exotic objects were created by a phase transition in the early universe and could exist over a variety of size and mass scales. If the strings are superconducting, as suggested, then their motion through a magnetized plasma could accelerate particles to high velocities and eventually produce X-radiation.

For the problem of dark matter in our galaxy, AXAF will be used to search for a variety of potential dark matter candidates. These studies include searches for the coronal X-ray emission from very low mass stars, for thermal X-ray emission, from the surfaces of isolated, cooling neutron stars, and for X-ray emission from isolated black holes accreting matter from the interstellar medium - all of which may be undetectable at other wavelengths, but are easily within the AXAF's capability.

The technique for probing dark matter in other galaxies is illustrated by the EINSTEIN observations of the giant elliptical galaxy M87 shown in Figure 3 . The emission extends over a region at least three times larger than that from which visible light has been detected. Such data can be converted to a profile of the gas density by using the fact that the X-ray intensity emitted by gas at low density scales as the square of the gas density. Together with a measure of the temperature profile, and under the reasonable assumption that the bulk of the gas is in hydrostatic equilibrium with the outward directed thermal pressure balanced by the inward directed gravitational force, one can compute the total mass interior to a given radius. When this was done for $\mathrm{M} 87$, for example, a mass profile was determined 
along with a total mass estimate of between 4 and $8 \times 10^{13} \mathrm{M}_{\odot}$. This is about 100 times more mass that determined from the visible light output of the galaxy, and also at least 30 times the mass seen in the X-ray emitting gas.

These measurements are unique in their use of the hot, X-ray emitting gas as a tracer to map the underlying gravitational potential or mass. In this manner, AXAF can measure the actual masses (including the dark matter content) of individual galaxies with hot gas in the outer regions as well as the masses of clusters of galaxies. Optical measurements to accomplish the same goals are not always feasible, often require numerous spectroscopic observations of individual tracer stars or galaxies, and may be greatly biased by local departures from isotropy and dynamical equilibrum. The X-ray data are not effected by these problems and therefore, in this particular endeavor, emphasize the uniqueness of X-ray observations. The EINSTEIN data demonstrated the feasibility of this approach, and AXAF will extend these studies to much fainter and more distant objects while also greatly improving the accuracy of surface brightness and temperature distributions. AXAF will determine masses to a precision of $10 \%$, an improvement of a factor of 10 over EINSTEIN.

\section{The Distance Scale of The Universe}

Another fundamental issue which AXAF will address is the distance scale of the universe, as well as its age and ultimate fate. A particularly novel and fundamental measurement of cosmic distances can be made by combining AXAF studies of clusters of galaxies with ground-based radio or microwave observations. This measurement hinges on the so-called Sunyaev-Zel'dovich effect. The effect arises when the cosmic microwave background radiation passes through the gas in a cluster of galaxies. The energetic electrons in the hot gas cloud scatter the photons, slightly increasing their temperature. The predicted change in microwave background temperature scales as (gas density) $\times$ (radius of gas cloud) $\times$ (gas temperature). The $\mathrm{X}$-ray observations will determine the luminosity [which scales as $\left(\right.$ density $\left.^{2}\right) \times\left(\right.$ radius $\left.\left.^{3}\right)\right]$, gas temperature, and angular size. The combined observations yield the cluster radius directly, and, from the angular radius, one can then determine the distance. AXAF data combined with already available microwave measurements would thus determine the distance to some clusters (e.g. A2218) to a precision of $30 \%$. Improvements in the microwave measurements already feasible and extension to millimeter wavelengths could conceivably improve this to better that $7 \%$

The Hubble Space Telescope will be used to measure distances with this order of precision, but on a relatively local scale. A2218, on the other hand, is at a distance comparable to that of many quasars, perhaps 50 times further away than HST will be measuring. At a minimum, AXAF will be able to apply this independent technique to determine the Hubble constant. Since the X-ray/microwave technique can, in principle, be extended to very large distances, AXAF has the potential to measure the Hubble constant as a function of distance. By looking at several clusters at distances corresponding to look-back-times of 10 billion years, AXAF could detect about a $20 \%$ change in the Hubble constant. This level of precision 
could thus distinguish between open and closed universes. Inflationary cosmological models involving an early phase of exponentially fast expansion, predict that the universe should be just at the boundary between open and closed. If supported by the AXAF observations, then a large amount of additional mass must be present in galaxies, in clusters, or in the voids of space.

\section{Stars to Quasars}

It is believed that stars, like planets, generate magnetic fields in their interiors with charged fluid motions. The primary AXAF observations in this area will involve the detection of X-ray emission from the outer coronae of thousands of stars, coupled with detailed X-ray spectroscopy for stars of varying temperatures and luminosities. The capability for performing such experiments was established by AXAF's prototype; EINSTEIN revealed the unexpected existence of hot X-ray emitting coronae around all types of stars. The conventional wisdom held that only stars with internal convectional processes like those of our Sun had the mechanisms to generate such coronae. Massive stars, too hot to have convection zones, were therefore not expected to have coronae and thus strong $\mathrm{X}$-ray emission. Theorists had to go back to the drawing board and have not yet returned. Stellar dynamo theories may be tested and improved by observing the correlations of coronal X-ray emission with stellar magnetic field strength, age, rotation, rate, and composition.

AXAF will study the evolution of high mass stars and probe the nature of the supernova process. The spatially resolved spectra of supernova remnants observed with AXAF should provide the data needed to distinguish the material ejected in the supernova explosion from the interstellar matter with which it has been mixed. With its sensitivity at relatively high energies, AXAF will be able to study the critically important iron lines. Combining this data with theoretical models will then help reveal the chemical composition of the progenitor star, its mass, and details about the nuclear reactions during the supernova event itself.

AXAF also will study the thermal radiation from neutron stars that have just been produced in supernova explosions and initially radiate in the $\mathrm{X}$-ray region of the spectrum. The observed rates of cooling of such neutron stars can be used as diagnostics to determine such parameters as size and thermal conductivity, which in turn depend on the equation of state of matter under extremely high densities and on general relativistic thermodynamics. Because of their enormously high density and gravity, neutron stars offer an excellent laboratory for the study of matter under extreme conditions and of the effects of general relativity. Indeed the contact with basic physics has been well established. The best current limit on the flux of magnetic monoples from space comes from EINSTEIN Observatory limits on neutron star surface temperatures which would be increased by nucleon decay catalized by magnetic monopoles, if present.

What can AXAF tell us about the formation and evolution of quasars? Due to the absence of substantial intergalactic absorption at $\mathrm{X}$-ray wavelengths and the ubiquitous association of $\mathrm{X}$-ray emission with quasars, AXAF can discover and study quasars at higher redshifts than any presently known or detectable with the 
Hubble Space Telescope. Such studies will determine the evolution of quasars over the longest time scales.

Related observations and deep surveys with AXAF are capable of determining the composition of the all-sky, cosmic X-ray background, thereby establishing the contribution of various classes of discrete sources to this background and setting a limit for any residual diffuse component. Since currently observed, broad-band $\mathrm{X}$-ray spectra of individual objects differ somewhat from the spectrum of the background, and theoretical calculations argue against a substantial diffuse component, these studies may discover new classes of astronomical objects or at least require modifications of present evolutionary scenarios.

\section{Conclusions}

AXAF is a member of the Great Observatories, which also include the Hubble Space Telescope, the Gamma-Ray Observatory, and the Space Infrared Telescope Facility. Operating in concert, and complementing each other in their respective wavelength capabilities, the Great Observatories should place mankind in a unique and historical position to understand the universe. It is of course presumptuous to claim that fundamental problems may be fully resolved by any new scientific mission. Indeed, the success of science is measured as much by the questions it raises as the questions it answers. In exploring such questions as those described above, AXAF has the potential to increase our knowledge at a fundamental level. With its state-of-the-art equipment for imaging and analyzing X-rays, AXAF will peer farther into space and farther back in time than any previous X-ray telescope. AXAF will help answer some of the most fundamental questions that have stirred the human mind: How far does space extend? Will the universe come to an end? What are the forces at work in the cosmos? What are the basic constituents of matter?

\section{Acknowledgements}

I wish to thank Alan Lightman, Harvey Tannanbaum, Steve O'Dell and the members of the AXAF Science Working Group. Special thanks to Paul Gorenstein, William Forman and Christine Jones for the Einstein Images.

\section{References}

Weisskopf, M.C., 1988, Astronomy and Astrophysics with the Advanced X-Ray Astrophysics Facility, Space Science Reviews, 47, pp. 47-93 